\title{
PLANEAMIENTO DEL TURISMO Y GEOGRAFÍA. DESARROLLO EN CUBA EN LOS ÚLTIMOS 40 AÑOS.
}

\author{
por
}

Estélez Pazó, R., Des Risco Yera, Y., SErRano RaFo, $F$.

(Facultad de Geografia, Universidad de La Habana, Instituto de Planificación Fisica)

\section{RESUMEN}

Cuba en la década de los años 90 , se ha visto precisada a desarrollar el turismo como vía de salida de la crisis económica en que se vio envuelta su economía. Sin embargo, la actividad no es nueva en el país. En el trabajo se aborda cómo ha sido el desarrollo del turismo en Cuba, fundamentalmente en los últimos cuarenta años, y los aportes que los estudios geográficos y de planificación han hecho al mejor desenvolvimiento de la misma.

PALABRAS Claves: turismo, planificación física, planificación turística.

\section{ABSTRACT}

Cuba in 90' decade, has been forced to developed the tourism as a means to improve to heal the serious situation of its economy. However, the activity is not new in the country. In the work it is approached like it has been the deve- lopment of the tourism in Cuba, fundamentally in the last forty years, and the contributions that the geographical studies and planning for the best development in the same one.

KEY WORDS: tourism, tourism management.

\section{EL TURISMO EN CUBA}

Quizás se pueda buscar la génesis del turismo en Cuba desde los mismos inicios del "encuentro entre las dos culturas"; o sea, desde la colonización del archipiélago cubano por los peninsulares.

La aparición del turismo en Cuba y su desarrollo han estado en estrecha correspondencia con la historia económica y político-social del país ( ER. SALINAS y Estévez R., 1996).

Durante el siglo XIX queda conformada la nacionalidad cubana. La 
Habana es una metrópoli de gran importancia, está consolidada urbanísticamente y cuenta con una cultura fuerte donde sobresale y se crea el danzón. Estas razones hacen que un gran número de visitantes norteamericanos arriben a la Isla por motivos turísticos y de negocios en los finales de este siglo.

En los primeros treinta años del siglo $\mathrm{XX}$, como producto del auge económico nacional, el turismo internacional en Cuba se incrementó (en lo fundamental, con visitantes norteamericanos). A esto coadyuva la creación en 1919 de la Comisión Nacional para el Fomento del Turismo; por lo que se afirma que, el surgimiento del turismo como industria, se produce en esta fecha (ER. SAlinAs y Estévez R., 1996).

Algunos investigadores (SERrano, Fr., 1997) han planteado que en esta época las estadísticas enmascaran las motivaciones reales de dichos viajes. En el caso cubano, el flujo estadounidense no sólo tenía motivaciones de ocio, sino que estaba relacionado con la penetración económica e, incluso, con las tentativas de colonización de territorios en Isla de Pinos.

En los años treinta, el turismo se vio afectado por la crisis de 1929; sin embargo, los viajes a Cuba implicaban obviar la Ley Seca, imperante en los EE.UU, lo que propició la aparición de un turismo vinculado al vicio y posteriormente al juego.
Después de la Segunda Guerra Mundial, se produce de nuevo un aumento de los viajes turísticos. Entre 1950 y 1958 arriban a la isla 1.857 .510 visitantes, siendo el año 1957 el de más visitantes (272.266), ( Villalba, Ev., 1993). Sin embargo, hasta la mitad de este decenio, la balanza de pagos registraba un déficit en el saldo turístico, debido a que la burguesía cubana, gastaba más dinero en el exterior, que el dejado por los turistas extranjeros en el país.

En fin, se puede afirmar que, a partir de 1930 y hasta 1958, Cuba fue el principal destino del Caribe, pero con un turismo poco sano, ligado en lo fundamental al vicio, al juego y a la prostitución. Es de destacar que el turismo doméstico era insignificante debido a la situación de subdesarrollo general del país.

En la década de los sesenta solamente arribaron a Cuba entre 3.000 y 5.000 visitantes extranjeros, como consecuencia, en primer lugar, de la política hostil de Estados Unidos y, luego, por la dependencia absoluta que este mercado tenía de los vecinos del Norte.

Sin embargo, durante este último periodo hay un cambio radical en la concepción del desarrollo del turismo, como reflejo del ideal socio-político que asumió la sociedad cubana. En 1959, la Ley 270 establece el libre acceso, sin distinción de personas, a todas la playas y 
íreas litorales. En ese año se crea con zarácter autónomo y personalidad jurídica propia el Instituto Nacional de la Industria Turistica ( INIT), cuyos objetiros básicos de trabajo eran (Salinas Er. ¿ EstÉvez R., 1996) los siguientes:

1. Programación de la política de fomento turístico.

2. Ejecución de los planes de desarrollo turístico.

3. Conservación y fomento de las riquezas naturales cubanas.

4. Protección del patrimonio histórico y cultural de la nación que sea relevante a los fines turísticos.

5. Promover la supresión progresiva de todas las causas del subdesarrollo turístico cubano.

En los años setenta se inicia una recuperación en la afluencia de turistas extranjeros a Cuba. Comienzan a llegar grupos organizados desde Canadá y de algunos países de América Latina, así como de Europa Oriental y Occidental. En la segunda mitad de esta década ocurrén tres hechos importantes: a) la creación, en 1976, del Instituto Nacional del Turismo (INTUR), con funciones de planificación y ejecución de la política de desarrollo turístico nacional y extranjero; b) el levantamiento de la prohibición de los ciudadanos cubanoestadounidenses de visitar a sus familiares en Cuba, lo que propició un importante flujo de visitantes, (esta situación acrecentó el interés por visitar el país; y c) en 1979, la VI Cumbre de los Paises no alineados, que comienza a promover el turismo de eventos científicos, sociales y congresos.

A finales de los años ochenta, el estado cubano concibe al turismo como elemento clave en la política de desarrollo y como actividad competitiva a nivel mundial. Como antecedente en 1982, se promulga el Decreto Ley 50, que regula la asociación económica entre entidades cubanas y extranjeras. Con la promulgación de esta Ley se estimula la asociación de empresas mixtas y sociedades cubanas con capital extranjero, y la explotación conjunta de instalaciones para el turismo. Así, se descentraliza el papel del INTUR como única organización administradora de turismo y, en 1987, surge la Corporación Cubanacán de Comercio Exterior y Turismo y, en 1988, el Grupo Gaviota SA (SALINAS ER. y EstÉveZ R., 1996).

Esta política se consolida en los años noventa donde hay un incremento sostenido de los indicadores turísticos y ocurre la última reestructuración de esta actividad, cuando se crea el Ministerio de Turismo, que tiene como objetivo principal establecer las políticas de desarrollo turístico, pero no administrar ninguna instalación. Aparecen las cadenas hoteleras Gran Caribe (hoteles de 5 y 4 estrellas), Horizontes (3 y 2 estrellas), Islazul, para 
atender al turismo nacional y el Grupo de Recreación y Turismo Rumbos. Todas estas empresas están facultadas para crear asociaciones con entidades extranjeras atendieñdo a la legislación vigente en Cuba (SALINAS Er. y EsTÉveZ R., 1996).

\section{PLANEAMIENTO DEL TURISMO EN CUBA. RESULTADOS Y PERSPECTIVAS}

El planeamiento en Cuba ha estado vinculado a su desarrollo como nación. Sin embargo, es a partir de las transformaciones socioeconómicas posteriores a 1959, cuando el ordenamiento territorial se imbrica en la dinámica del desarrollo como un poderoso agente de cambio, portador de directivas y lineamientos, que posibilitan -de acuerdo a las circunstancias particulares del momento histórico- el desarrollo equilibrado de una determinada actividad. En el caso específico del turismo, la concreción de este planeamiento reviste características particulares, asociado a las especificidades de la llamada "industria del ocio"; de ahí, que su expresión sea compleja, porque se relaciona en el territorio con otras actividades económicas y sociales. Éstas permiten vincular metodológicamente al turismo con el planeamiento (SERRANo F., 1998), lo que garantiza que la actividad turística no sea la causante de la destrucción del medio ambiente del que se sirve para garantizar su propia existencia como actividad lucrativa, ya que
"(...) el crecimiento incontrolado del turismo puede destruir irremediablemente la misma base de recursos sobre el que fue cimentado (PEARCE, 1994).

Es a partir de 1959, cuando el planeamiento físico comienza a ocupar su lugar como herramienta certera en el desarrollo del turismo. Se pueden distinguir dos grandes etapas del planeamiento físico del turismo en Cuba:

a) La primera, que abarca las décadas de los años 60-70 y parte de los años 80 , del siglo XX; se caracteriza por concretar en todo el territorio nacional las aspiraciones de equidad social, a partir de la proclamación de Cuba como estado socialista, que posibilitó un cambio sustantivo en la expresión urbanística; los criterios de localización y de acceso a las instalaciones turísticas por parte de la población, no sólo de las principales ciudades del país, sino también de otras regiones y localidades menos favorecidas en el pasadó.

Durante los años 70 el planeamiento ya contaba con cierta experiencia que le permitió estudiar los principales territorios con potencialidades turísticas. En este contexto se realiza por el Instituto de Planificación Física, en 1974, el "Estudio para una Propuesta Nacional de Turismo", cuyo objetivo central era determinar la capacidad potencial del país para satisfacer las expectativas del vacacionista nacional; si bien se incluyó una propuesta de uso internacional de algunos 
erritorios que se consideraban satisfacorios para su explotación (razonaniento consecuente con una proyec:ión a mediano plazo, característica del slaneamiento físico) dadas las condicioıes físico-geográficas, ambientales, de nfraestructura y demográficas del trchipiélago cubano.

b) A finales de la década de los 80 , 1 Estado Cubano elabora las directivas sara convertir el turismo en un sector :lave de la economía. A su vez, el planetmiento del turismo había experimenado un salto cualitativo con el reforzaniento del Sistema de la Planificación Física (SPF), (SERrano, 1998), para así esponder a la ejecución de planes de lesarrollo y dar respuesta a las crecienes inversiones en el sector. La actualiza:ión de la documentación normativa zompletaba la base conceptual que recesitaba la actividad del planeamieno para imprimirle, al ordenamiento erritorial, una dinámica consecuente zon las peculiaridades de la actividad urística contemporánea.

La creación del MINTUR (Miniserio del Turismo) propició en 1994 la zlaboración de la tarea "Política de Desarrollo de la Actividad Turistica en Cuba", y se le asignó al Sistema de la Planificación Física la determinación del Potencial Natural Turistico, cuyo objetivo Zundamental era: "definir, en colaboración con las instituciones correspondientes, los botenciales de los polos y regiones del país en cuanto a recursos naturales, histórico-culturales, cientifico-técnicos, infraestructuras ingenieras y fuerza de trabajo". (Instituto de la Planificación Física, IPF, 1995). Esta tarea y otras semejantes, enriquecen y formulan políticas de desarrollo territorial del Esquema Nacional de Turismo, en fase de realización, cuyos resultados posibilitan establecer las directivas en diferentes horizontes temporales: situación actual, año $2.000 \mathrm{y}$ pleno desarrollo.

Es conveniente resaltar que durante el desarrollo del planeamiento del turismo en Cuba en los últimos 40 años del siglo, (en consonancia con el nivel de conocimiento alcanzado en cada una de las etapas anteriormente descritas) el Sistema de Planificación Física ha propiciado una estrecha vinculación con la protección del medio ambiente y el uso racional de los recursos naturales, respaldada por los diversos documentos legales (leyes, decretos, normas, entre otros) que han sido promulgados en estos años y que permiten dirimir cualquier contradicción que se presente entre el desarrollo del sector turístico y la conservación del patrimonio natural y cultural de la nación.

En este sentido, reviste especial importancia la reciente promulgación de la Ley 81 del Medio Ambiente de la República de Cuba, que actualiza la política ambiental del país con un enfoque de desarrollo sostenible, y establece 
pautas para la actividad turística y el planeamiento sobre bases sostenibles.

\section{POTENCIAL NATURAL TURÍSTICO}

Uno de los elementos sobresalientes y de mayor connotación en el planeamiento del turismo lo constituye el conocimiento del potencial natural del área que se desea desarrollar. En este sentido, varios son los estudios que durante los últimos años se han realizado en Cuba; algunos de ellos de alcance nacional. Tarea ardua para los especialistas del turismo pues, al margen de las premisas y consideraciones que se utilicen para enmarcar el universo a estudiar, no es menos cierto que las condiciones naturales de Cuba satisfacen las expectativas de un gran número de modalidades turísticas. En relación con las playas, elemento dimensionador por excelencia del segmento turístico más sobresaliente en la actualidad, se plantea que existen más de 300 (la mayor parte en estado natural) con parámetros de longitud, ancho de franja de sol, calidad de la arena y pendiente, que satisfacen las expectativas del turismo internacional $y$, por ende, la localización de instalaciones hoteleras y extrahoteleras.

La riqueza de la flora, la vegetación, la fauna y la diversidad de sus paisajes constituyen un patrimonio de gran envergadura en Cuba. De acuerdo con el criterio de especialistas, Cuba ocupa el $5^{\circ}$ lugar en el planeta en cuanto al número de endémicos de su flora vascular natural (IPF, 1997). En este sentido, la propuesta para integrar un Sistema de Áreas Protegidas que abarque cerca del $10 \%$ de la superficie emergida del país, constituye un potencial para la práctica del turismo de naturaleza. Lo anterior ha servido de base para la elaboración de inventarios de recursos turísticos naturales, cuyo universo estará en dependencia de la tendencia de los mercados y las características de los territorios, siendo el soporte para posteriores clasificaciones.

El país también cuenta con otros potenciales importantes para desarrollar el turismo, entre los que pueden citarse los histórico-culturales, socioeconómicos, y las infraestructuras. Aunque el turismo de playa es muy importante, como producto de su desarrollo histórico, es significativa la importancia del turismo de ciudad en cuanto a la capacidad hotelera ( playas: $58 \%$ y ciudad: $31,8 \%$ de la capacidad total, en 1995), (SERRANo, F., 1998).

En resumen, se puede afirmar que el clima, la estructura geologo-geomorfológica y la insularidad son los tres factores físico-geográficos que definen y caracterizan el potencial turístico-recreativo de los recursos naturales de Cuba ( SAlinas Er. y ESTÉveZ R., 1996). 


\section{IRSPECTIVAS DEL DESARROLLO DEL URISMO EN CUBA}

En las previsiones de la OMT para el io 2.000 , la región caribeña recibirá J millones de visitantes y diez años desués recibirá 30 millones (periódico ranma, 1997). Dentro de esta prefesncia por la región, Cuba debe ser un estino importante. En el período 1990395 la tasa de crecimiento anual del ırismo fue del $17 \%$ en el número de sitantes y del $35 \%$ en los ingresos, asando de 340.300 visitantes en 1990, 741. 000 en 1995 y de 243,4 millones e dólares de USA, a 1.100 millones en 995. En estos años el turismo creó 0.000 puestos directos de trabajo y tros tantos indirectos, es decir, de uno or uno ( MINTUR, 1997). En el año 997, el número de visitantes fue de .170 .100 visitantes, lo que significa un recimiento del 16,5 \% con relación a 996 (ONE, 1998), y en 1998, la cifra egó a 1.400.000 (periódico Granma, 2/1/99). Para mantener e incremenur estos crecimientos y que Cuba contiúe posicionándose dentro de la egión, la actividad turística requiere er cada vez más competitiva, por lo que $s$ imprescindible continuar diversifiando su producto turístico, incremenar la calificación del personal vinculao al turismo y perfeccionar su política e desarrollo. Por ello, el Ministerio de urismo, junto a diferentes instituciones el país, ha elaborado su política para os próximos años.

\section{POLÍTICA TURÍSTICA}

Ante todo se trata de establecer el Potencial Turistico de la Isla. Los estudios realizados han permitido establecer 85 polos turísticos y un potencial de alojamiento de aproximadamente 153.000 habitaciones sujetas a fuertes restricciones para conservar el medio ambiente: promedio de 40 habitaciones por hectárea y preservar el $75 \%$ del área libre de edificaciones, así como no levantar más de cuatro plantas en instalaciones hoteleras, excepto en las ciudades (Granma, 1997). Así se delimitaron 16 regiones turísticas, de ellas 8 se clasificaron como principales: La Habana, Varadero, Norte de Ciego de Avila, Norte de Camagüey, Norte de Holguin, Sur de Oriente, Costa Sur del Centro y Archipiélago de los Canarreos.

Dentro de esta política se le da particular importancia al estudio de los mercados y su diversificación, a la infraestructura, a las fuentes de financiación (dónde, cuándo y cómo incrementar habitaciones) garantizando el crecimiento continuo de la participación de los productos nacionales en los insumos al turismo que, en el año 1997, fue del $41 \%$ frente al 35\% en 1996 (Granma, 1998). Para cumplir con esto, en 1998 el MINTUR aportó un financiamiento de alrededor de 80 millones de dólares (MINTUR, 1997).

La proyección del turismo cubano para el año 2.000, tiene dos aspectos fundamentales: 
- La consolidación de Cuba como destino en el Caribe y el mundo.

-Importante crecimiento en visitantes e ingresos (la proyección con tres escenarios):

a) Menor crecimiento, debido a trabas migratorias a cubano-americanos: 1.500 .000 visitantes y 45.000 habitaciones.

b) Mercados tradicionales + nuevos mercados + turistas cubano americanos: 2.500 .00 visitantes y 50.000 habitaciones.

c) El escenario b), más la eliminación de las trabas a los ciudadanos norteamericanos a visitar a Cuba: 2.800.000 visitantes y 75.000 habitaciones.

Según los criterios de los especialistas, el escenario b) es el más factible. Aún así se debe trabajar arduamente para lograrlo y como dijera CARLos LAGE, Secretario Ejecutivo del Consejo de Ministros, "El turismo se tiene que poner planes muy duros, en primer lugar, por la necesidad que tenemos de buscar los recursos para enfrentar la situación financiera $y$, en segundo lugar; porque estamos convencidos de que existen las posibilidades de que el país puede mejorar los indicadores económicos" (Granma, 1998).

\section{LOS ESTUDIOS DE TURISMO EN LA FACULTAD DE GEOGRAFÍA DE LA. UNIVERSIDAD DE LA HABANA}

El tema del turismo siempre ha estado presente dentro de las líneas de investigación de la Facultad de Geografía. El objetivo central de estos estudios ha sido descubrir las influencias territoriales de la actividad turística, ya que el tema de las relaciones espacioturismo no ha sido lo suficientemente estudiado en el país. La Facultad en sus investigaciones no concibe al turismo como un sector o rama de la economía, sino como una actividad con múltiples aristas (económicas, sociales, culturales y ambientales, entre otras).

Los primeros trabajos realizados fueron, sobre todo, estudios de evaluación de potenciales naturales para la determinación de áreas con interés turístico. Se trabajaron zonas como los archipiélagos que bordean la isla mayor y la región del norte de las Tunas y Camagüey, entre otras.

Ya en los años ochenta, apoyándose en las metodologías de ordenamiento geoecológico y territorial, se realizaron propuestas de zonificaciones funcionales, trabajando de forma conjunta con organismos de planificación, entre los que se pueden citar los estudios integrales de los grupos insulares y zonas litorales del archipiélago cubano con fines turísticos, algunos de los cuales se presentan ahora en esta publicación.

Los estudios más recientes han visto profundizar el ámbito de las aplicaciones. Son dignas de destacar las investigaciones encaminadas a valorar el potencial cubano para diversificar su producto turístico y desarrollar modali- 
ades alternativas. Dentro de éstos se ueden mencionar los trabajos en la ona del Nicho, en la provincia de ienfuegos; el Saltón en la provincia de antiago de Cuba y en Cayo Coco para roponer su utilización dentro de ıdalidades de ecoturismo. Aquí se an diseñado y propuesto senderos de xploración que se convierten en opcioales a ser tomadas muy en cuenta. Así lismo, también se puede mencionar el studio de las posibilidades de desarroar el turismo rural en áreas como el ıunicipio de Trinidad y el análisis de is limitaciones para ello.

También se ha trabajado en la valoxción de impactos ambientales produidos por el desarrollo de proyectos urísticos (Cayo Las Brujas), así como n el diseño de productos turísticos mbientalmente compatibles con paisas sensibles, como en el caso de Cayo aguanes. Para ello, ha sido un valioso poyo la experiencia acumulada en los studios geoecológicos, como base de la lanificación ambiental en paisajes senbles, que son casi siempre los más emandados por el turismo. Se han ropuesto lineamientos a ser tomados n cuenta en la planificación y gestión el turismo, por ejemplo en el caso del rchipiélago de los Canarreos. También ? han desarrollado trabajos de diseño e Sistemas de Información Geográfica para su uso en el planeamiento del turismo en diversos territorios: Cienfuegos-Trinidad y Archipiélago de los Canarreos, entre otros.

En las ciudades se han inventariado los recursos del patrimonio históricocultural y sus relaciones con la demanda. También se han realizado aproximaciones a los complejos temas de las relaciones espacio-turismo en las ciudades.

De vital importancia ha sido la impartición, a partir de 1995 del programa de maestría "Geografia, Medio Ambiente y Ordenamiento Territorial" que cuenta con la mención de "Turismo y Ordenamiento Territorial" que ya cuenta con graduados sobre la temática.

El proyecto cubano ha cifrado grandes esperanzas en el turismo como motor impulsor de la economía. Pero para que esto se produzca de la forma más idónea, de manera que reporte los resultados económicos esperados, sin producir impactos ambientales negativos, es necesario continuar con estudios cada vez más científicos que, con una visión objetiva, conlleven a peldaños superiores del desarrollo. En este sentido la Facultad de Geografia continuará encaminando sus estudios, junto a otras instituciones vinculadas a la temática, nutriéndose de las experiencias acumuladas en estos años transcurridos. 